\title{
Chemistry in Augmented Reality
}

\author{
Sanket Salve ${ }^{1}$, Akshay Khapare ${ }^{2}$, Rohit Barve ${ }^{3}$ \\ Final Year Students, Department of Information Technology, Vidyalankar Institute of Technology, Mumbai, India ${ }^{1,2}$ \\ Assistant Professor, Department of Information Technology, Vidyalankar Institute of Technology, Mumbai, India ${ }^{3}$
}

\begin{abstract}
The comprehension of atom-world has always been the focus and the challenge of chemistry learning. Junior high school student's imaginative abilities are not yet mature. As a result, they are not able to visualize structures correctly during the beginning stage of chemistry learning. The Augmented Reality application of Chemistry in AR has its own features, teaching information capacity, interactive user interface. Through Augmented Reality, an application will be developed which will help the students to easily visualize the 3D structure of an element or a molecule. Students could control, combine and interact with a 3D model of micro-particles using markers. It will also help the student to visualize and understand the chemical reactions by combination of two or more elements or molecules. The chemistry modelling and spatial arrangement of molecular structures in space can be understood using 3D objects. It enables user to interact with virtual and real world in real time application.
\end{abstract}

Keywords: Augmented Reality, Unity 3D, Vuforia SDK, Multimedia Technology, Chemistry learning.

\section{INTRODUCTION}

For many learners across the world, chemistry is introduced for the first time in junior high school. Abstract concepts such as molecules, atoms, and amount of substance are formidable to junior high school students. These students are often required to envision across microand macro-worlds, which can be extremely challenging. "The composition of substances" is a critical concept in chemistry learning, as it is the foundation of further learning about chemicals and organic chemistry. However, young students' imaginative abilities are limited, and it is difficult for them to imagine how particles such as atoms compose substances. This problem necessitates improvement in the learning methods and tools used in chemistry teaching. Augmented Reality (AR) is an extension of Virtual Reality (VR). By contrast to traditional VR, AR provides a seamless interface for users that combines both the real world and the virtual world. Users can interact with virtual objects that are interposed on real scenes around them and obtain the most natural and genuine human-computer interaction experience. Only an Android smartphone with a camera will be needed to construct a local AR environment. The camera detects markers within its vision and then presents the scene it captures and the corresponding virtual objects represented by the markers simultaneously on the mobile screen. Users can move the markers to interact with the interposed virtual objects. Users can get information about the physical properties of the elements on demand. By moving two markers close enough, users can also perform chemical reactions by combining two elements or molecules which will help them to easily visualize it.

\section{PREVIOUS WORK}

There are various websites and web apps over the internet which helps students to study the elements and their reactions. Students have a problem in learning and understanding structures of elements andmolecules. In current scenario, they learn from 2D representations like blackboard and book. It is a very tedious task to learn and memorize the atomic structure of elements. The 3D structure of elements and molecules cannot be visualized and understood by the students easily.

El Sayed, Zayed, and Sharawy (2011) devised an Augmented Reality Student Card (ARSC), which can represent any lesson in a 3D format that aids students in visualizing different learning objects, interact with theories and manage information in a totally new way. The research suggests that ARSCs increase students' visualization abilities using a minimum number of tools. Nunez, Quiros, Nunez, Carda, and Camahort (2008) presented an AR system for teaching spatial relationships and chemical problems with university-level students. In the experiment, students could manipulate crystal structures of certain substances, such as $\mathrm{ZrSiO} 4$ with markers. However, in the studies above, only static images or structures are rendered.

Through Augmented Reality, the 3D chemistry modelling and spatial arrangement of molecular structures in space can be understood. It enables user to interact with virtual and real world in real time application. Students will be able to easily visualize and understand the structures of elements and molecules and they can also visualize and understand the chemical reactions by combination of two or more elements or molecules.

\section{OVERVIEW}

The Android application in Augmented Reality will provide ways to learn and visualize the structure of chemical elements and molecules. Spatial ability plays an important role in chemistry learning, as students are 
required to visualize specific microstructure, but the A. Reading Input

visualization of microstructure is difficult task for The camera sensor will be activated and it will search for students. Using Augmented reality in chemistry, students the image target which is placed on the marker which in will get to understand, comprehend, interpret, and translate this case would be the element, molecule or reaction name. molecular representation.This Android application will be used by professors as well as students and can also be used by other users to visualize and understand the structures of elements and molecules. User can also perform the chemical reactions by combination of two or more elements or molecules. The application will contain elements from s-block, p-block, d-block elements. When two elements will be brought close to each other a molecule formation will occur.The application will have login for every user and will have different user-interfaces for users like Professor, Student and other users. This application will be managed by an administrator who can make changes to the database. This is a standalone application which is compatible with Android operating system. Thus, the application will help the students to visualize and understand the structure of element and molecules as it will be depicted in a 3D-representaion model.

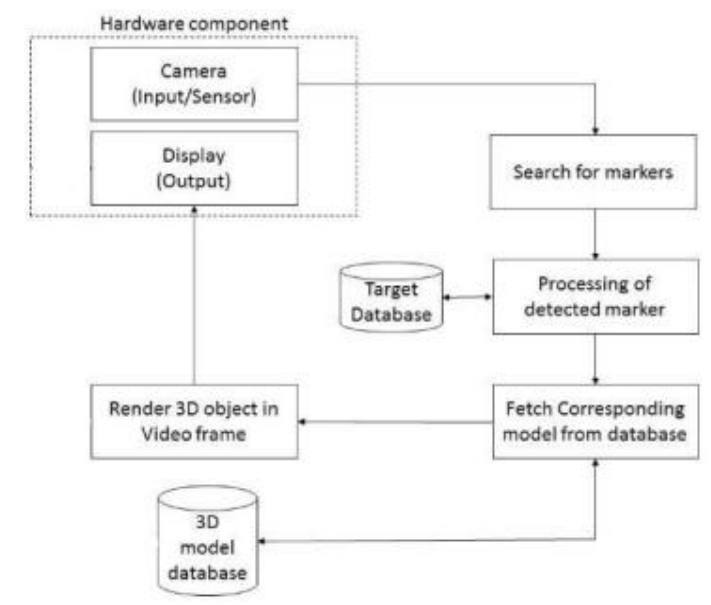

Fig.1. Proposed System Diagram

\section{METHODOLOGY}

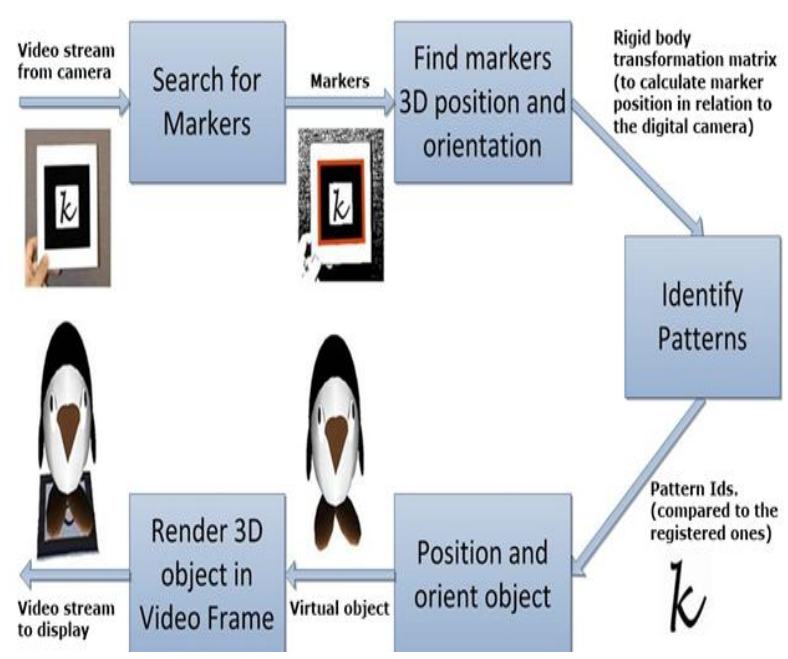

Fig 2. Methodology
B. Find Position

In this step, process of finding markers position and 3D position and orientation will initiate.

C. Conversion and Identifying pattern

The image is then converted into image transformation matrix and the pattern is matched with pattern in database.

After matching patterns, the object corresponding to that pattern of the marker which will be a 3D element or molecule will be retrieved from the database.

\section{Mapping}

The position of virtual object retrieved from the database after pattern matching is mapped relative to camera and displayed relative to marker (The image target). This object will be visible to the user in a 3 dimension and it will be visible as long as the marker is present

Next, the application will check for user interactions with the markers ie. formation of a molecule or occurrence of a chemical reaction which will happen when two markers will be brought close to each other. Again after the actions the object will be retrieved from the database and will be displayed on the marker.

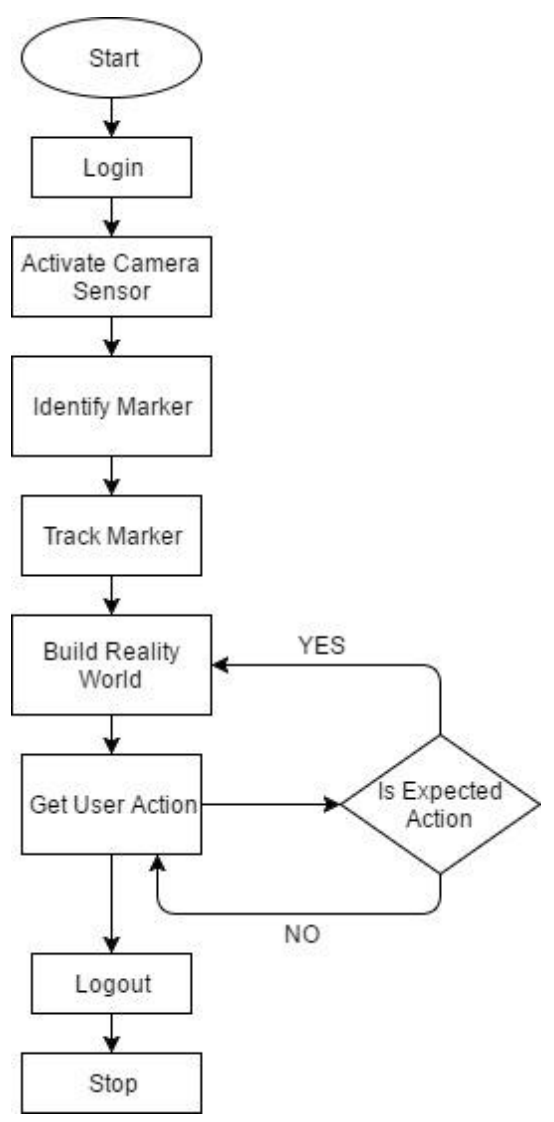

Fig.3. Process Flow Chart 


\section{CONCLUSION}

The project will help the students to visualize the structure in 3-Dimension and understand the structure of elements, structure and formation of different molecules and help to understand the chemical reactions between molecules easily with the help of Augmented Reality which provides a seamless interaction between real and virtual environment. This project will be a great help for the students as they can learn without visiting the laboratory.

\section{REFERENCES}

[1] https://www.computer.org/csdl/proceedings/3dui/2006/0225/00/022 50135-abs.html

[2] https://pdfs.semanticscholar.org/5a78/12e7109c013e5b07c9b104c4 f943405554be.pdf

[3] http://ceur-ws.org/Vol-91/paperE4.pdf

[4] http://www.sciencedirect.com/science/article/pii/S07475632140022 71

[5] http://file.scirp.org/Html/1-9301927_48585.htm

[6] http://www.divaportal.org/smash/get/diva2:663778/FULLTEXT01.pdf

[7] http://ir.lib.uwo.ca/cgi/viewcontent.cgi?article=3271\&context=etd

[8] http://dl.acm.org/citation.cfm?id=1128990

[9] https://capstone.cs.ucsb.edu/team_docs_13/SRS/SRS_HexPistols.p df

[10] https://www.theseus.fi/bitstream/handle/10024/45551/Markus_Lius ka.pdf?sequence $=1$

[11] http://homepages.cwi.nl/ paulk/theses/VanDerZee.pdf 